\title{
Diferenciais Salariais Inter-regionais, Interindustriais e Efeitos Fixos Individuais: Uma Análise a Partir de Minas Gerais ${ }^{\star}$
}

\author{
- Ricardo da Silva Freguglia* - Naercio A. Menezes-Filho** \\ - DenIS BARRETo de SOUZA***
}

\section{RESUMO}

Este artigo tem como objetivo identificar se os diferenciais de salário entre os trabalhadores de diferentes regiões do Brasil e de diversos ramos industriais persistem após o controle pelas características não-observáveis destes indivíduos, que são fixas ao longo do tempo. Para atingir este objetivo, usamos a base de dados da Raismigra (MTE) para acompanhar os trabalhadores que estavam formalmente empregados na indústria de transformação de Minas Gerais entre 1999 e 200I, usando a abordagem de dados em painel. Os principais resultados obtidos indicam que os diferenciais de salário entre regiões e entre ramos de atividade persistem após o controle pela heterogeneidade dos trabalhadores, mas sua importância diminui significativamente.

\section{PalavRAS-CHAVE}

diferenciais salariais, migração, dados em painel, indústria mineira

\begin{abstract}
The aim of this paper is to examine whether the inter-regional and inter-industry wage differentials in Brazil persist after controlling for the non-observable workers characteristics that are fixed over time. We use data from Raismigra (Labor Ministry) to follow workers that were formally employed in Minas Gerais industry between 1999 and 200 I using a panel data approach. The main results show that the interregional and inter-industry wage differentials persist after controlling for individual-specific fixed effects, but lose importance.
\end{abstract}

\section{KEY WORDS}

wage differentials, migration, panel data, industry of Minas Gerais

\author{
JEL CLASSIFICATION \\ $J 3 I, J 6 I$
}

\footnotetext{
+ Os autores agradecem ao Ministério do Trabalho e Emprego pelo fornecimento dos dados utilizados e aos valiosos comentários do parecerista da revista Estudos Econômicos.

* Doutorando IPE/USP e Professor FEA/UFJF-Universidade Federal de Juiz de Fora. Bolsista Capes. Endereço para contato: R. Djalma Dutra, 649 - ap. 104 - Vila Ocidental Presidente Prudente - SP - CEP: 190I5-040. E-mail: ricardo.freguglia@ufjf.edu.br.

* Professor do Departamento de Economia do IPE/USP e do IBMEC-SP. E-mail: NaercioAMF@isp.edu.br.

$\star \star \star$ Mestrando em Economia da UFRGS - Universidade Federal do Rio Grande do Sul. E-mail: denis@fecap.br. (Recebido em março de 2005. Aceito para publicação em maio de 2006).
} 


\section{INTRODUÇÃO}

Um fato estilizado na literatura econômica relativa à estrutura salarial é a existência de grandes e persistentes diferenciais salariais entre ramos de atividade para trabalhadores de mesma qualificação e ocupação. (Dickens e Katz, 1987; Krueger e Summers, 1988). Tais disparidades permanecem mesmo após o controle por diferentes atributos observáveis dos indivíduos e do emprego, que afetam a utilidade dos trabalhadores. Neste sentido, os diferenciais de salários entre os ramos de atividade parecem ser uma característica comum de uma economia de mercado que, entretanto, as teorias competitivas não conseguem explicar.

Uma explicação alternativa é que os diferenciais salariais observados são um reflexo de diferenças não observadas na qualidade do trabalhador. Estudos recentes, como o de Carruth, Collier e Dickerson (2004), ressaltam que as equações de salários em cross-section explicam menos da metade das variaçóes salariais e que existem grandes e persistentes diferenciais de salário interindústrias. Os autores concluem que a adição de efeitos não observados explica cerca de $90 \%$ da variação salarial na Inglaterra e que os prêmios salariais por indústria, apesar de pequenos, permanecem estatisticamente significativos após a inclusão destes efeitos.

Tendo em vista que os diferenciais salariais no Brasil têm sido durante muito tempo objeto de atenção devido à grande desigualdade de renda que caracteriza o País, a identificação da verdadeira natureza e da fonte de diferenciais salariais observados entre indústrias torna-se uma questão de grande relevância. Arbache e De Negri (2004), por exemplo, examinam os efeitos da filiação industrial - vínculo empregatício de um trabalhador em uma firma do setor industrial - sobre os salários no Brasil, controlando por variáveis como o tamanho das plantas, nacionalidade do capital e acesso ao mercado internacional, além de variáveis associadas aos trabalhadores. Os resultados encontrados evidenciam que as diferenças nos salários têm na filiação industrial um importante componente explicativo.

No que se refere ao aspecto regional da desigualdade de renda, o quadro geral continua sendo de grandes e persistentes diferenciais salariais. Tomando como base os indicadores de salário per capita dos trabalhadores, as diferenças entre as regiões Sul/ Sudeste e Norte/Nordeste, além de significativas, evidenciam um enorme contraste: são os moradores destas últimas que recebem os menores salários, o contrário acontecendo com os residentes no Sul/Sudeste. (Azzoni e Menezes, 2001). Buscando avaliar o papel das características do trabalhador e do emprego na explicação da desigualdade regional no Brasil, Azzoni e Servo (2002) analisaram a desigualdade salarial entre as dez maiores regióes metropolitanas no Brasil nos anos 1990. Os principais resultados indicam que os diferenciais salariais no Brasil são reduzidos quando estes controles 
de características do trabalhador e do emprego são introduzidos, mas a desigualdade ainda permanece elevada.

Este artigo avança na abordagem destes tópicos ao analisar explicitamente a função da heterogeneidade não observada como uma explicação dos diferenciais salariais observados entre indústrias e regiôes, sendo possível estimar mais detalhadamente os efeitos individuais. Mais especificamente, este estudo examina a magnitude dos diferenciais salariais entre os trabalhadores de diferentes regiões do Brasil e entre diversos ramos industriais após o controle pelas características não observáveis destes trabalhadores que são fixas ao longo do tempo. Para atingir este objetivo, usamos dados longitudinais da Raismigra - MTE -, e diante do grande volume de observaçóes disponíveis focamos a análise no Estado de Minas Gerais nos anos de 1999 e 2001. Os dados possibilitam examinar evidências em cross-section, mínimos quadrados agrupados e efeitos não observados dos diferenciais salariais entre ramos de atividade e regiões.

O trabalho está organizado em seis seções além desta introdução. Na primeira seção apresentamos as consideraçóes teóricas sobre os diferenciais salariais interindustriais e inter-regionais e a heterogeneidade não observada. A segunda seção detalha a fonte de informações utilizada neste estudo e a terceira seção faz uma breve caracterização da migração inter-reginal e interindustrial dos trabalhadores da indútria de transformação. A seção quatro descreve a metodologia de dados em painel. As evidências econométricas encontradas são objeto de análise da quinta seção. A última seção conclui o trabalho.

\section{DIFERENCIAIS SALARIAIS INTERINDUSTRIAIS, INTER-REGIONAIS E HETEROGENEIDADE NÃO OBSERVADA}

Os principais estudos sobre o padrão de diferenciais salariais interindústrias e interregião ressaltam o elevado grau de estabilidade, no tempo, destas disparidades, que parecem se manter entre uma variedade de países com distintos arranjos institucionais e estruturais. (Gittleman e Wolff, 1993; Kahn, 1998). Elas também persistem entre diferentes tipos de trabalhadores e tamanhos de estabelecimentos, mesmo após o controle por diferenças no capital humano, ocupação e outras variáveis. (Krueger e Summers, 1988). Estes resultados sugerem que os diferenciais salariais são compatíveis com o funcionamento das economias capitalistas, questionando a validade das teorias competitivas para explicar a dispersão de salários.

Como ressalta Fernandes (2002), as fontes da desigualdade salarial podem estar associadas às diferenças dos trabalhadores em relação às suas características produtivas e preferências e/ou às imperfeições de mercado que impedem a mobilidade dos traba- 
lhadores dos postos de trabalho com baixos salários para aqueles com altos salários. Trata-se de uma questão de ênfase: de um lado estão aqueles que acreditam que as imperfeições de mercado desempenham um papel importante na desigualdade de salários e, de outro, aqueles que acreditam que o mercado de trabalho funciona relativamente bem e diferenças em habilidades e preferências dariam conta de explicar a maior parte da desigualdade salarial observada.

Segundo os modelos competitivos do mercado de trabalho, mudanças na dispersão salarial deveriam ser altamente transitórias por natureza. Na teoria do capital humano tradicional, os atributos do emprego que não afetam diretamente a utilidade dos trabalhadores não deveriam ter efeitos na determinação dos salários individuais. Em contraste, as teorias não competitivas de determinação salarial postulam que tais atributos podem ter um efeito sistemático sobre os salários porque influenciam o salário ótimo para as firmas se ajustarem, implicando mudanças permanentes. O propósito dessas teorias é determinar por que as firmas podem achar lucrativo pagar salários maiores que a taxa de equilíbrio de mercado e por que a importância destes fatores difere por indústria.

De um modo geral, a investigação da determinação de salários e do diferencial de salários em países desenvolvidos tem se concentrado nos efeitos do capital humano e nas diferentes formas de segmentação associadas aos arranjos institucionais e características estruturais dos rendimentos, como aquelas associadas à discriminação, rent-sharing (divisão de rendas/lucros) e sindicatos. No caso dos países em desenvolvimento, porém, a investigação dos diferenciais de salário tem focado, primeiramente, a contribuição das variáveis de capital humano para os rendimentos e secundariamente sobre os fatos estilizados de que o mercado de trabalho é segmentado entre os setores moderno e tradicional, formal-informal, público-privado e empresas de propriedade estrangeira-nacional. Além disso, os efeitos restritivos da legislação trabalhista, salários-mínimos e sindicatos também afetam a dispersão salarial, enquanto as teorias competitivas e de segmentação explicadas pelos modelos de salário-eficiência têm recebido menos atenção como uma fonte de variação salarial.

No Brasil, os diferenciais de salário têm sido objeto de atenção devido à grande desigualdade de renda que caracteriza o País. A explicação para esta questão ganha relevância pelo fato de que, mesmo quando controlados por uma série de características observáveis, como educação, idade, região de residência, ocupação etc., estes diferenciais persistem. Uma possibilidade é que tais diferenciais reflitam características produtivas não observáveis.

Neste sentido, uma explicação alternativa que oferece novos elementos para a análise do diferencial de salários no Brasil é que existem diferenças não observadas na 
qualidade do trabalhador que causam este diferencial. A hipótese de habilidade não mensurada se baseia no fato de que os empregadores escolhem os trabalhadores não apenas por suas habilidades que podem ser medidas, como educação e experiência no trabalho, mas também por suas habilidades não observadas. Como estas não são, por definição, diretamente captadas pelos dados, grande parte das características produtivas dos trabalhadores deixa de ser considerada nas estimativas econométricas padrão. (Arbache, 2001).

Carruth, Collier e Dickerson (2004) mostram a importância da heterogeneidade não observada na determinação do salário. Segundo os autores, cerca de $90 \%$ da variação dos rendimentos dos trabalhadores da indústria na Inglaterra pode ser explicada por características individuais observadas e não observadas. Porém, pequenos - mas estatisticamente significantes - retornos salariais na indústria permanecem.

O objetivo deste estudo é investigar o papel da heterogeneidade não observada como uma explicação para os diferenciais observados entre indústrias e regiões. Utilizamos dados em painel e a metodologia de efeito fixos para mostrar, explicitamente, que muito dos diferenciais salariais observados entre indústrias e regiões em cross-section podem ser explicados por efeitos individuais específicos observados e não observados.

\section{DADOS}

A base de dados utilizada é a RAISMIGRA, do MTE - Ministério de Trabalho e Emprego -, que é derivada dos registros administrativos da RAIS - Relação Anual de Informações Sociais. Seu objetivo central é o acompanhamento longitudinal da trajetória profissional dos trabalhadores segundo as características geográficas, setoriais e ocupacionais, permitindo a realização de estudos de mobilidade do indivíduo no mercado de trabalho. A principal diferença em relação à RAIS, portanto, é que a RAISMIGRA está organizada de modo a identificar cada trabalhador ao final de cada ano da trajetória, possibilitando a caracterização do próprio trabalhador, do posto de trabalho por ele ocupado e do vínculo empregatício. Isto não é possível com o uso da RAIS convencional, que está organizada por ano de referência da declaração dos vínculos. (MTE, 2003).

Sendo originária da RAIS, o universo de análise também se restringe aos trabalhadores formalmente empregados. Portanto, os problemas decorrentes da natureza da obtenção dos dados da RAIS, cujas informações sobre os trabalhadores são reportadas pelas empresas, também se encontram presentes na RAISMIGRA. As possíveis distorções dos dados podem gerar um viés da amostra na presença de erros de medida. Em virtude da organização longitudinal dos dados, os erros de medida são potencia- 
lizados, especialmente em níveis mais desagregados das informações, como município e ocupação, por exemplo.

Neste estudo, a amostra utilizada para os anos de 1999 e 2001 considerou os indivíduos com vínculo empregatício na indústria de transformação da região metropolitana de Belo Horizonte - RMBH - e renda positiva, formando um painel balanceado com um total de 280.138 observações (140.069 indivíduos). Assim, podemos analisar os diferenciais salariais interindustriais e intersetoriais dos trabalhadores que saíram da indústria de transformação da região metropolitana de Belo Horizonte, considerando tanto os trabalhadores migrantes quanto os não-migrantes. $\mathrm{O}$ migrante é aquele que muda de setor de 1999 para 2001 (no caso da migração intersetorial) e o nãomigrante é o que permanece no mesmo setor em 2001 comparativamente a 1999. O mesmo ocorre na migração inter-regional. Estão presentes na amostra, portanto, os trabalhadores que trocaram de emprego. Entretanto, não temos como acompanhar longitudinalmente os trabalhadores que se tornaram inativos ou informais.

As variáveis utilizadas foram renda, grau de instrução, idade, sexo, experiência, localização geográfica e setor de atividade. ${ }^{1}$ No que se refere à escolaridade, foram utilizadas as oito categorias que compõem as etapas do ciclo educacional, conforme disponibilizado pelo MTE na Raismigra. A experiência se refere ao tempo de emprego do trabalhador, em meses, no mesmo vínculo de emprego. Os setores de atividade foram definidos conforme a classificação de atividades econômicas do IBGE - Instituto Brasileiro de Geografia e Estatística: administração pública, agropecuária, construção civil, comércio, indústria extrativa mineral, indústria de transformação, serviços industriais de utilidade pública e setor de serviços.

\section{EVIDENNCIAS INICIAIS}

O objetivo desta seção é caracterizar, de forma incipiente, a migração inter-regional e interindustrial dos trabalhadores da indústria de transformação da região metropolitana de Belo Horizonte entre os anos de 1999 e 2001. Esta apresentação permite dimensionar o fenômeno em questão e traçar o perfil médio dos trabalhadores migrantes, fornecendo os subsídios iniciais para a compreensão dos resultados das estimativas desenvolvidas nas etapas seguintes deste estudo.

Considerando inicialmente a migração inter-regional, 6.209 trabalhadores migraram para outras regiões/Estados do País, correspondendo a $4,5 \%$ do total da amostra. ${ }^{2}$

1 Algumas inconsistências encontradas nos dados relativos a estas variáveis foram eliminadas por meio da exclusão da observação com erro de medida.

2 Os indivíduos que não migraram entre regiões perfazem um total de 133.860 trabalhadores. 
No total de migrantes, o destino da maioria foi a saída de Minas Gerais para outro Estado (52\%), com destaque para São Paulo, com 23,5\%. Os demais migraram para o Rio de Janeiro (7\%), Região Sul (6,5\%), Nordeste (6\%), Centro-Oeste (5\%), Espírito Santo (3\%) e Norte (1\%). Por sua vez, a migração para o interior do próprio Estado de Minas Gerais também foi expressiva, especialmente para a região central do Estado mineiro, que abrange os municípios no entorno da Região Metropolitana de Belo Horizonte (22\%), e para o sudeste do Estado (12\%).

A Tabela 1 descreve o perfil da migração inter-regional de acordo com a escolaridade, sexo, idade, experiência e renda do trabalhador. De modo geral, o migrante, isto é, aquele trabalhador formalmente empregado na indústria de transformação que mudou da região metropolitana de Belo Horizonte em 1999 para outra região ou Estado em 2001 possui, em média, pouco mais de 32 anos, trabalha no mesmo emprego há aproximadamente 16 meses e tem uma renda de R $1.128,52$. São homens em sua grande maioria $(81 \%)$, como era de se esperar, tendo em vista que os trabalhadores considerados são originários da indústria de transformação, e o nível de escolaridade mais representativo é o $2^{\circ}$ grau incompleto.

Tais características são úteis na descrição dos migrantes, mas ganham relevância quando comparadas com a dos não-migrantes. Estes últimos são mais velhos que os primeiros (1 ano a mais, em média), são representados por um maior porcentual de homens ( 5 pontos porcentuais a mais) e possuem uma experiência bem mais elevada, quase 40 meses superior à dos migrantes. No que se refere ao nível de escolaridade e a renda média, os não-migrantes são menos escolarizados e a sua renda é inferior à dos migrantes em quase R\$300,00. É interessante notar que, no caso da migração para o interior do Estado mineiro, o diferencial de renda é negativo. Tal evidência pode estar refletindo as diferenças no custo de vida entre estas regiões. Em geral, estes dados mostram que os Estados/regiões de maior renda média, como São Paulo e Rio de Janeiro, ou mais próximos da região de origem, como a região central e o sudeste do Estado, são os que mais absorvem os trabalhadores migrantes.

Na migração intersetorial, 34.399 indivíduos, correspondendo a 24,5\% do total da amostra, ${ }^{3}$ tiveram como destino outro setor diferente da indústria de transformação. Os setores que mais absorveram trabalhadores foram os serviços e o comércio que, juntos, representavam $86 \%$ do total de migrantes. Em seguida estão os setores de construção civil $(9,53 \%)$, administração pública $(2,43 \%)$, agropecuária $(1,58 \%)$, extrativa mineral $(0,66 \%)$ e os serviços industriais de utilidade pública $(0,24 \%)$.

3 Os indivíduos que não saíram da indústria de transformação somam 105.670 trabalhadores. 


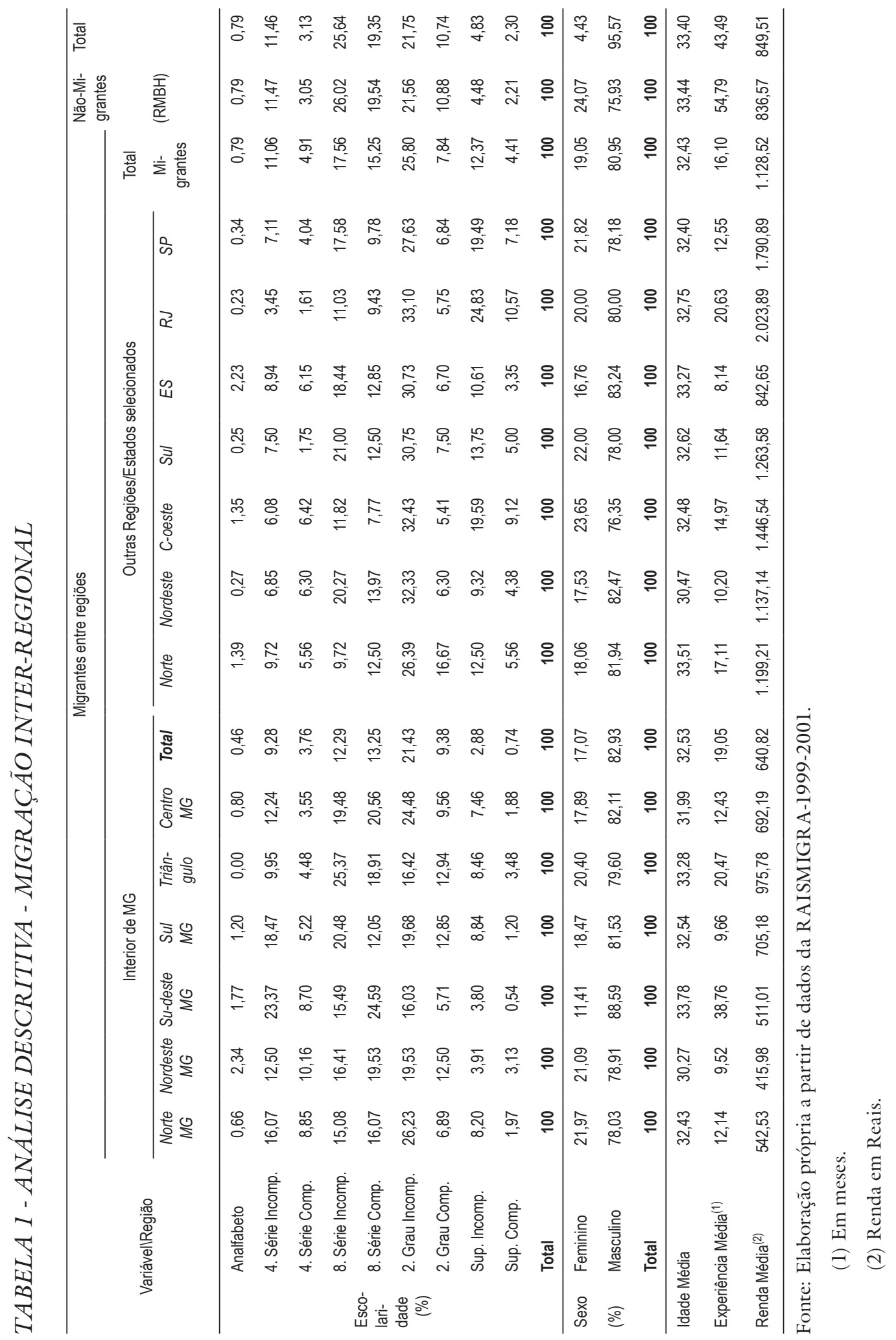


Pela Tabela 2 podemos verificar que os migrantes são três anos mais jovens (31 anos) que os não-migrantes e possuem uma experiência no mesmo vínculo empregatício bastante inferior. Salta aos olhos a migração em direção à construção civil, cujos trabalhadores têm apenas 8,5 meses de experiência no mesmo emprego. Isto é um reflexo da alta rotatividade existente no setor.

As mulheres representam $28 \%$ do total de migrantes, porcentual que supera em 5 pontos a participação feminina dentre os não-migrantes. Dentre os setores de destino dos migrantes, o predomínio masculino é marcante nas atividades de construção civil, extrativa mineral, serviços industriais de utilidade pública e agropecuária. Este predomínio é relativizado quando a migração ocorre em direção aos setores de administração pública, comércio e serviços, nos quais a participação feminina é de 40\%, $35 \%$ e $27 \%$, respectivamente.

É interessante notar que os não-migrantes com curso superior completo representam um porcentual maior que o de migrantes na mesma categoria (5,1\% contra 4,0\%). Porém, o porcentual de migrantes é superior ao de não migrantes com $2^{\circ}$ grau (completo ou incompleto) e superior incompleto. No que se refere à remuneração dos trabalhadores que mudam de setor, estes possuem uma renda média (R \$ 626) inferior à dos não-migrantes ( $\mathrm{R}$ \$ 922), revelando indícios de que o trabalhador possui custos no processo de transição intersetorial.

De modo geral, os dados apresentados evidenciam uma forte concentração da migração para o Estado de São Paulo e a região central do Estado de Minas Gerais e para os setores de serviços e comércio. Os migrantes são mais jovens e menos experientes, em média, comparativamente aos não-migrantes em ambas as dimensões da análise da migração: inter-regional e intersetorial. Apesar da maior participação masculina, as mulheres migram mais entre os setores que entre regiões. No tocante à escolaridade e à renda, os migrantes são mais instruídos que os não-migrantes na dimensão interregional e a renda média é superior em relação ao outro grupo. Na dimensão intersetorial, os migrantes são, em geral, mais instruídos, exceto na faixa de trabalhadores com nível superior completo. A renda, ao contrário da situação verificada na dimensão inter-regional, é inferior, em média, àquela dos trabalhadores não-migrantes. 


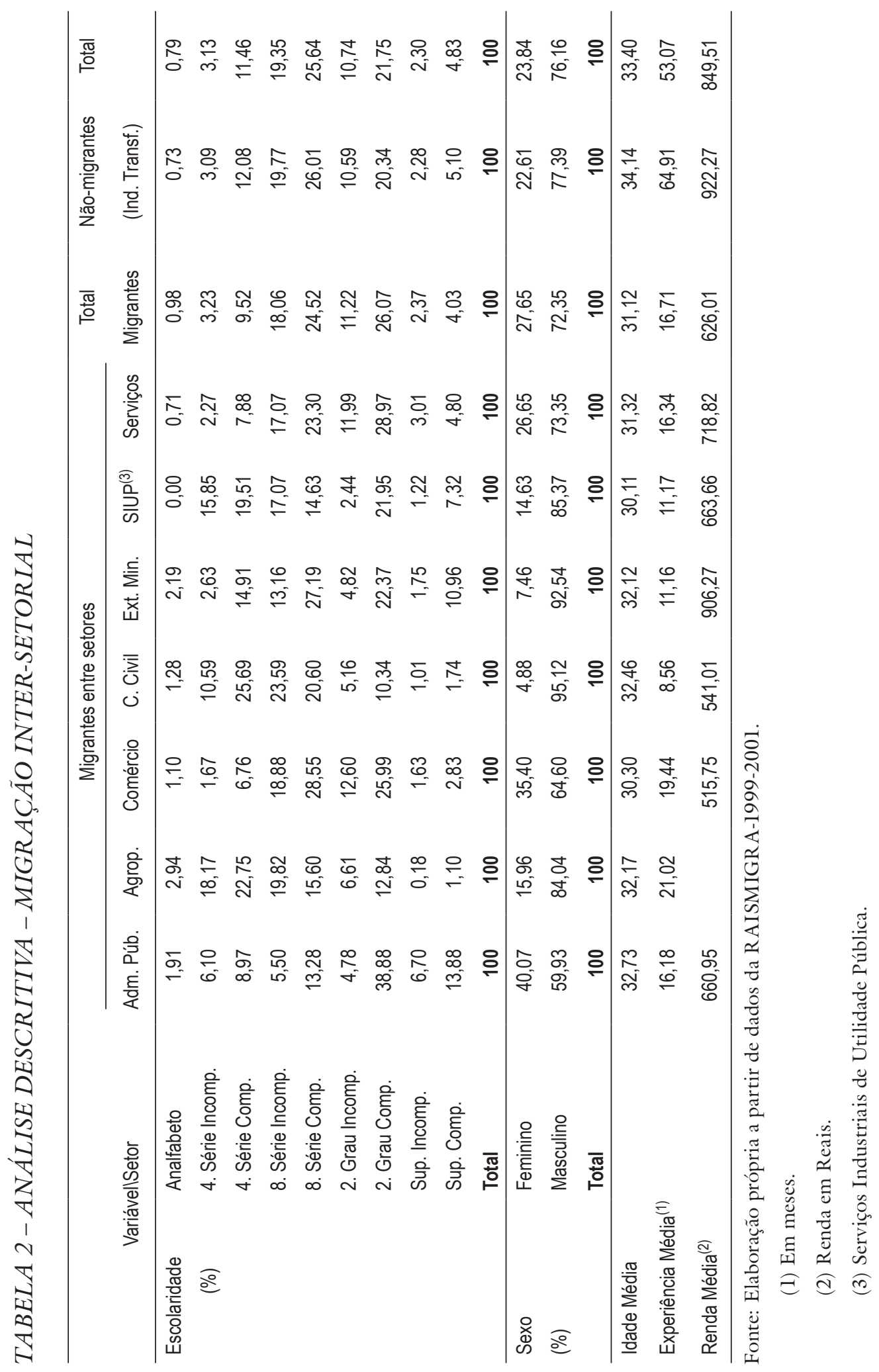




\section{METODOLOGIA ECONOMÉTRICA}

Usando os dados longitudinais para os anos de 1999 e 2001, estimamos as equações de rendimentos para os trabalhadores migrantes da indústria de transformação da região metropolitana de Belo Horizonte. Para avaliar os efeitos da mobilidade regional e setorial do trabalhador, bem como as características do trabalhador na determinação da renda, estimamos as regressões em nível ${ }^{4}$ para os dois anos considerados, 1999 e 2001. A forma funcional destas regressões segue o padrão de cortes transversais destacado pela literatura (Menezes-Filho, 2002; Corseuil e Santos, 2002), tendo como variável dependente o logaritmo da renda e como variáveis de controle a idade, idade ao quadrado, experiência, experiência ao quadrado, dummies de grau de instrução e dummy de sexo, subsumidas no vetor $\mathrm{X} ; \delta_{i}$ são os diferenciais de renda associados às dummies inter-regionais $\left(R_{i}\right) ; \theta_{j}$ representam os diferenciais de renda relacionados às dummies interindustriais $\left(I_{i}\right)$ e $\varepsilon_{i}$ é o termo de erro aleatório (equação (1)).

$$
\log \left(\text { renda }_{i}\right)=\alpha+\beta X_{i}+\sum_{i} \delta_{i} R_{i}+\sum_{j} \theta_{j} I_{j}+\varepsilon_{i}
$$

Em seguida, buscamos a definição do modelo mais adequado aos dados e ao objetivo central do estudo. Inicialmente, estimamos a equação de renda por mínimos quadrados agrupado, que considera as observações para os anos de 1999 e 2001 indistintamente, como se fosse um grande corte transversal. Apesar de ser um método freqüentemente utilizado em estudos como este, seu principal problema está relacionado à validade da hipótese de que não há informação de $\varepsilon_{i}$ que esteja correlacionada com as variáveis explicativas:

$$
E\left(\varepsilon_{i} \mid x_{i}\right)=0 ; E\left(\varepsilon_{i} \mid R_{i}\right)=0 ; \quad E\left(\varepsilon_{i} \mid I_{i}\right)=0
$$

A relação de causalidade, portanto, ocorre somente com a manutenção desta hipótese. Caso contrário, surge o problema de endogeneidade, gerando estimadores inconsistentes e viesados. Como, em geral, não podemos observar o potencial de auferir rendimentos do trabalhador, possivelmente existirá uma correlação destas características não observáveis - como a habilidade, capacidade de relacionamento, adaptação a mudanças, criatividade, motivação, dentre outras - com alguns dos determinantes dos salários - em especial a escolaridade, região de domicílio, ocupação - invalidando a interpretação causal dos coeficientes estimados.

No caso da mobilidade de trabalhadores da indústria de transformação de Minas Gerais, a escolha da região ou do setor por parte do trabalhador está correlacionada com o seu potencial de rendimentos, ou seja, dado que uma regiáo (indústria) espe-

4 A correção da variância não se fez necessária, pois as estimativas robustas de mínimos quadrados não apresentam mudanças significativas no erro padrão. 
cífica tende a remunerar melhor as habilidades não observadas, o trabalhador com essas características será encaminhado para esta região (indústria). Assim, não se pode afirmar com certeza que um trabalhador com determinadas características irá obter ganho ou perda salarial ao se mover de uma região (indústria) para outra.

Uma das maneiras de se lidar com o problema de endogeneidade é o método de efeitos fixos. Como existem dados sobre os migrantes e não-migrantes antes e depois da migração (dados da Raismigra em painel), podemos incluir um efeito fixo na regressão, que vai captar todas as características não observadas dos trabalhadores que são constantes ao longo do tempo (c):

$$
\log \left(\text { renda }_{i t}\right)=\alpha+\beta X_{i t}+\delta_{1} R_{i t}+\delta_{2} I_{i t}+c_{i}+\varepsilon_{i t}
$$

A hipótese de identificação ${ }^{5}$ do modelo requer agora que $E\left(\varepsilon_{i} \mid c_{i}, R_{i}, I_{i}\right)=0$, ou seja, que toda a correlação entre $R_{i}$ e $I_{i}$ e $\varepsilon_{i}$ seja captada por uma covariada, que não varia entre períodos. A estimação poderia ser feita após a inclusão de uma dummy para cada trabalhador, por mínimos quadrados ordinários ou por primeiras diferenças. ${ }^{6}$ Nesse último caso, a heterogeneidade não observada seria automaticamente eliminada. Cabe ressaltar que é necessário que uma parte da amostra mude de região e de indústria ao longo dos anos considerados para que os coeficientes dos diferenciais salariais entre indústrias e regiões possam ser identificados, após a inclusão dos efeitos fixos.

Além disso, é importante observar que o uso de efeitos fixos pode não ser suficiente para eliminar o viés de variável omitida, pois, conforme Menezes-Filho (2001), a decisão de migrar poderia estar correlacionada com fatores omitidos que provocariam uma alteração na renda esperada dos indivíduos. Como exemplo, podemos considerar o caso em que os trabalhadores que migraram (ou mudaram de indústria) foram "incentivados" por uma redução transitória de renda real. Na análise de primeiras diferenças, o aumento de renda seria imputado à migração (ou mudança de indústria), quando o que de fato aconteceu foi uma regressão para a média. O problema é que os estimadores de primeiras diferenças e de efeitos fixos não são robustos à inclusão de uma variável dependente defasada. Uma possível solução seria utilizar na análise somente os trabalhadores que mudaram de setor involuntariamente, devido ao fechamento de seus locais de trabalho, por exemplo. Esta análise, contudo, foge do escopo

5 A hipótese de identificação é que a migração é aleatória, ou seja, não se baseia em características não observadas que mudam no tempo. Como a análise é de diferencial de salários inter-regionais e intersetoriais, para controlar pelo efeito fixo (heterogeneidade não observada) dos trabalhadores, precisamos comparar os salários dos trabalhadores que migraram com o dos que não migraram por meio de um modelo de efeitos fixos.

6 Como consideramos apenas dois períodos no tempo, 1999 e 2001, o método de primeiras diferenças possui estimativas e estatísticas de teste idênticas às do método de efeitos fixos. Por isso, optamos por apresentar somente a análise de efeitos fixos. 
deste trabalho. A seção seguinte apresenta os principais resultados obtidos com as estimações realizadas.

\section{RESULTADOS}

Inicialmente analisamos as regressões em nível para os anos de 1999 e 2001. Pela Tabela 3 observa-se que os diferenciais salariais entre as regiôes do Estado de Minas Gerais, com exceção da região do Triângulo Mineiro, são negativos, ou seja, os trabalhadores que se movem para outras regiões ganham menos renda do que ganhariam se tivessem permanecido na região metropolitana de Belo Horizonte. Isto pode estar refletindo diferenças relativas ao custo de vida existente entre a região metropolitana e as cidades do interior.

Em contraste, os diferenciais salariais dos trabalhadores que se movem para fora do Estado são todos positivos. Para ilustrar este fato, podemos verificar que a renda dos migrantes para as regiões de São Paulo e Rio de Janeiro foi 42,77\% e 40,24\% (respectivamente) superior à renda dos trabalhadores que não migraram. $\mathrm{O}$ mesmo ocorre para as demais regiões do País, porém com porcentuais inferiores. Estes resultados da mobilidade inter-regional, particularmente na migração interestadual, evidenciam a seleção positiva dos migrantes, isto é, os migrantes possuem características não observadas - habilidade, motivação, determinação, perseverança etc. - que os diferenciam dos não-migrantes em termos salariais, formando um grupo positivamente selecionado. É válido notar, contudo, que os coeficientes das variáveis da região do sul de Minas Gerais e da região do Espírito Santo não são significativos.

Na migração entre os setores de atividade, observamos que, com exceção do setor da indústria extrativa mineral, todos os migrantes apresentaram uma renda menor em relação aos trabalhadores que permaneceram na região metropolitana de Belo Horizonte. Uma possível explicação está relacionada à difícil adaptação de um trabalhador que troca de setor de atividade, o que faria com que ele não conseguisse manter o mesmo padrão de rendimento do setor de origem. No caso dos trabalhadores que migraram para a indústria extrativa mineral, estes apresentaram uma renda 10,07\% superior aos trabalhadores que permaneceram na indústria de transformação. 
TABELA 3 - REGRESS ÃO EM NÍVEL - REGIÃO METROPOLITANA DE BELO HORIZONTE - VARIÁVEL DEPENDENTE: LOGARITMO DA RENDA

\begin{tabular}{|c|c|c|c|c|c|}
\hline \multirow[t]{2}{*}{ Regressor } & \multicolumn{2}{|c|}{1999} & \multicolumn{3}{|c|}{2001} \\
\hline & Coef. & Erro Padrão & Coef. & & Erro Padrão \\
\hline Constante & 3,508 & 0,020 & 3,843 & * & 0,024 \\
\hline \multicolumn{6}{|c|}{ Características do trabalhador } \\
\hline Idade & 0,093 & 0,001 & 0,079 & * & 0,001 \\
\hline Idade $^{2}$ & $-0,001$ & 0,000 & $-0,001$ & * & 0,000 \\
\hline Sexo - masculino & 0,472 & 0,003 & 0,477 & * & 0,003 \\
\hline Experiência & 0,006 & 0,000 & 0,005 & * & 0,000 \\
\hline Experiência $^{2}$ & 0,000 & 0,000 & 0,000 & * & 0,000 \\
\hline \multicolumn{6}{|l|}{ Nível educacional } \\
\hline Analfabeto & (excluída) & & & & \\
\hline Ensino médio incompleto & 0,308 & 0,014 & 0,364 & * & 0,017 \\
\hline Ensino médio completo & 0,632 & 0,014 & 0,640 & * & 0,017 \\
\hline 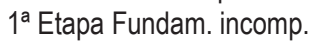 & $-0,151$ & 0,016 & $-0,126$ & * & 0,019 \\
\hline $1^{\text {a }}$ Etapa Fundam. comp. & $-0,074$ & 0,014 & $-0,023$ & & 0,017 \\
\hline 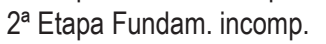 & 0,052 & 0,014 & 0,080 & * & 0,017 \\
\hline 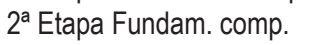 & 0,175 & 0,014 & 0,232 & * & 0,017 \\
\hline Superior incompleto & 1,167 & 0,017 & 1,185 & * & 0,019 \\
\hline Superior completo & 1,628 & 0,015 & 1,698 & * & 0,018 \\
\hline \multicolumn{6}{|l|}{ Localização } \\
\hline Região Metropolitana BH & (excluída) & & & & \\
\hline Norte de MG & - & & $-0,158$ & * & 0,032 \\
\hline Nordeste de MG & - & & $-0,291$ & * & 0,049 \\
\hline Sudeste de MG & - & & $-0,281$ & * & 0,020 \\
\hline Sul de MG & - & & $-0,047$ & & 0,035 \\
\hline Triângulo mineiro & - & & 0,135 & * & 0,039 \\
\hline Centro de MG & - & & $-0,061$ & * & 0,015 \\
\hline Região Norte & - & & 0,183 & * & 0,068 \\
\hline Região Nordeste & - & & 0,177 & * & 0,030 \\
\hline Espírito Santo & - & & 0,070 & & 0,041 \\
\hline Rio de Janeiro & - & & 0,402 & * & 0,026 \\
\hline São Paulo & - & & 0,428 & * & 0,015 \\
\hline Região Sul & - & & 0,247 & * & 0,027 \\
\hline Região Centro Oeste & - & & 0,291 & * & 0,033 \\
\hline Setor de atividade & - & & & & \\
\hline Adm. Pública & - & & $-0,276$ & * & 0,019 \\
\hline Agropecuária & - & & $-0,223$ & * & 0,024 \\
\hline Comércio & - & & $-0,224$ & * & 0,005 \\
\hline Construção Civil & - & & $-0,123$ & * & 0,010 \\
\hline Extrativa Mineral & - & & 0,101 & * & 0,036 \\
\hline Ind. Transformação & (excluída) & & & & \\
\hline SIUP & - & & $-0,156$ & * & 0,061 \\
\hline Serviços & - & & $-0,065$ & * & 0,005 \\
\hline
\end{tabular}

Número de observações: 140.069

Fonte: Elaboração própria a partir de dados da RAISMIGRA-1999-2001.

* Significativo a $5 \%$. 
Além dessas evidências, a Tabela 3 traz uma série de resultados que, embora secundários em relação ao objetivo central do estudo, merecem destaque. Os homens ganharam, em média, $47,5 \%$ a mais que as mulheres em 1999, porcentual que teve um ligeiro decréscimo em 2001, passando para 46,7\%. Para a idade, observamos que o logaritmo da renda do trabalhador cresceu a taxas decrescentes em ambos os anos, sendo que a idade de maior rendimento ficou em 45 anos em 2001. No que se refere à experiência, esta apresentou elevada influência na determinação do logaritmo da renda, crescendo a taxas decrescentes em ambos os anos.

O trabalhador que possui superior completo ganhou, em média, 162,75\% a mais do que um trabalhador analfabeto em 1999, enquanto que em 2001 esse porcentual foi de 169,78\%; houve um aumento do retorno da educação em todos os níveis de escolaridade entre 1999 e 2001. A diferença dos rendimentos entre níveis de escolaridade aumentou entre 1999 e 2001, ou seja, a diferença de renda entre um trabalhador com curso superior completo e de um trabalhador com curso médio completo era, em 1999, de 157,39\%, passando para 165,43\% em 2001.

É importante observar que a análise em nível não permite que controlemos características dos trabalhadores que não sejam observadas, mas que afetam a variável explicada renda. Assim, usaremos a metodologia de dados em painel que possibilita controlar os efeitos específicos do trabalhador, considerando que tais efeitos sejam invariantes em relação ao tempo.

A Tabela 4 apresenta os resultados de nossas estimativas para os modelos de mínimos quadrados agrupados, efeitos aleatórios e efeitos fixos. A primeira coluna reporta os coeficientes do modelo de mínimos quadrados agrupados que são significativos ao nível de 5\%, com exceção da região sul de Minas Gerais e do Estado do Espírito Santo. Entretanto, tais coeficientes devem ser enviesados e inconsistentes, pois podem estar incluindo características não observáveis, tais como aptidão, motivação, empreendedorismo, habilidade, entre outras, que devem estar relacionadas com as variáveis explicativas do modelo.

Portanto, com o objetivo de medir a importância potencial desta heterogeneidade não observada, estimamos um modelo de efeitos fixos usando os dados em painel da Raismigra considerando dois pontos no tempo: 1999 e 2001. No entanto, para decidirmos pelo método de estimação mais adequado utilizamos, primeiramente, o teste de Breusch-Pagan, ${ }^{7}$ que verifica a existência de efeitos não observáveis. O teste rejeita a hipótese nula da ausência de efeitos específicos a $1 \%$ de significância. Diante da existência de efeitos específicos, usamos o teste de Hausman, que rejeita a hipótese nula - também a $1 \%$ de significância - de que a diferença entre os estimadores de efeitos aleatórios e os de efeitos fixos seja pequena. Isto indica que o melhor modelo a ser utilizado é o de efeitos fixos e, desta forma, detalharemos a seguir a análise deste modelo.

$7 \quad$ Ver Wooldridge (2002, cap. 10). 
TABELA 4 - ESTIMATIVA DE DIFERENCIAIS DE RENDA DOS TRABALHADORES DA REGIÃO METROPOLITANA DE BELO HORIZONTE ENTRE 1999 E 2001 - VARIÁVEL DEPENDENTE: LOGARITMO DA RENDA

\begin{tabular}{|c|c|c|c|c|c|c|c|c|}
\hline \multirow[t]{2}{*}{ Regressor } & & \multicolumn{2}{|c|}{ MQO (agrupado) } & \multicolumn{3}{|c|}{ Efeitos Aleatórios } & \multicolumn{2}{|c|}{ Efeitos Fixos } \\
\hline & & Coef. & $\begin{array}{l}\text { Erro } \\
\text { padrão }\end{array}$ & Coef. & & $\begin{array}{l}\text { Erro } \\
\text { padrão }\end{array}$ & Coef. & $\begin{array}{l}\text { Erro } \\
\text { padrão }\end{array}$ \\
\hline \multirow{2}{*}{\multicolumn{2}{|c|}{$\begin{array}{l}\text { Constante } \\
\text { Ano } 2001\end{array}$}} & 3,604 & 0,015 & 3,657 & * & 0,017 & 6,004 & 0,002 \\
\hline & & 0,113 * & 0,002 & 0,120 & * & 0,001 & 0,210 & 0,001 \\
\hline \multicolumn{9}{|c|}{ Características do trabalhador } \\
\hline Idade & & $0,866 *$ & 0,001 & 0,091 & * & 0,001 & \multirow{2}{*}{\multicolumn{2}{|c|}{ (excluída - efeito fixo) }} \\
\hline Idade $^{2}$ & & $-0,001 *$ & 0,000 & $-0,001$ & * & 0,000 & & \\
\hline \multicolumn{2}{|l|}{ Sexo - masculino } & $0,475 *$ & 0,002 & 0,467 & * & 0,003 & \multirow{2}{*}{0,004} & \\
\hline \multirow{2}{*}{\multicolumn{2}{|c|}{$\begin{array}{l}\text { Experiência } \\
\text { Experiência }^{2}\end{array}$}} & $0,006 *$ & 0,000 & 0,005 & * & 0,000 & & 0,000 \\
\hline & & $0,000 *$ & 0,000 & 0,000 & * & 0,000 & 0,000 & 0,000 \\
\hline \multicolumn{9}{|l|}{ Nível educacional } \\
\hline Analfabeto & & (excluída) & & & & & & \\
\hline Ensino médio incompleto & & 0,333 * & 0,011 & 0,197 & * & 0,010 & & \\
\hline Ensino médio completo & & $0,633 *$ & 0,011 & 0,436 & * & 0,010 & & \\
\hline $1^{\text {a }}$ Etapa Fundam. incomp. & & $-0,140 *$ & 0,012 & $-0,131$ & * & 0,011 & & \\
\hline $1^{\text {a }}$ Etapa Fundam. comp. & & $-0,052$ & 0,011 & $-0,075$ & * & 0,010 & \multirow{2}{*}{\multicolumn{2}{|c|}{ (excluída - efeito fixo) }} \\
\hline $2^{\mathrm{a}}$ Etapa Fundam. incomp. & & $0,065 *$ & 0,011 & 0,011 & & 0,009 & & \\
\hline $2^{\mathrm{a}}$ Etapa Fundam. comp. & & 0,201 * & 0,011 & 0,098 & * & 0,009 & & \\
\hline Superior incompleto & & $1,173 *$ & 0,013 & 0,879 & * & 0,012 & & \\
\hline Superior completo & & 1,662 * & 0,012 & 1,382 & * & 0,011 & & \\
\hline & \\
\hline \multirow{6}{*}{ Interior de MG } & Norte de MG & $-0,151$ * & 0,031 & $-0,155$ & * & 0,023 & $-0,161$ & 0,024 \\
\hline & Nordeste de MG & $-0,284 *$ & 0,048 & $-0,253$ & * & 0,036 & $-0,248$ & 0,038 \\
\hline & Sudeste de MG & $-0,278 *$ & 0,020 & $-0,172$ & * & 0,015 & $-0,148$ & 0,015 \\
\hline & Sul de MG & $-0,361$ & 0,341 & $-0,878$ & * & 0,025 & $-0,116$ & 0,027 \\
\hline & Triângulo mineiro & 0,142 & 0,038 & $-0,060$ & * & 0,028 & $-0,181$ & 0,030 \\
\hline & Centro de MG & $-0,052$ & 0,015 & $-0,115$ & * & 0,011 & $-0,167$ & 0,011 \\
\hline Região Norte & & 0,188 & 0,067 & 0,105 & * & 0,050 & 0,060 & 0,052 \\
\hline Região Nordeste & & 0,182 & 0,029 & 0,131 & * & 0,022 & 0,119 & 0,023 \\
\hline Espírito Santo & & 0,759 & 0,040 & 0,020 & & 0,030 & 0,011 & 0,031 \\
\hline Rio de Janeiro & & $0,407 *$ & 0,026 & 0,020 & & 0,019 & 0,096 & 0,020 \\
\hline São Paulo & & $0,435 *$ & 0,014 & 0,262 & * & 0,011 & 0,166 & 0,011 \\
\hline Região Sul & & 0,257 & 0,027 & 0,100 & * & 0,020 & 0,023 & 0,021 \\
\hline Região Centro Oeste & & $0,300 *$ & 0,032 & 0,143 & * & 0,024 & 0,032 & 0,025 \\
\hline \multicolumn{9}{|l|}{ Setor de atividade } \\
\hline Adm. Pública & & $-0,262$ & 0,019 & $-0,158$ & * & 0,014 & $-0,030$ & 0,015 \\
\hline Agropecuária & & $-0,212$ & 0,024 & $-0,136$ & * & 0,018 & $-0,101$ & 0,019 \\
\hline Comércio & & $-0,209$ & 0,005 & $-0,130$ & * & 0,004 & $-0,066$ & 0,004 \\
\hline Construção Civil & & $-0,102$ * & 0,010 & $-0,057$ & * & 0,007 & $-0,068$ & 0,008 \\
\hline $\begin{array}{l}\text { Extrativa Mineral } \\
\text { Ind. Transformacão }\end{array}$ & & $\begin{array}{c}0,116 \\
\text { (excluída) }\end{array}$ & 0,036 & 0,148 & * & 0,027 & 0,151 & 0,028 \\
\hline SIUP & & $-0,141$ * & 0,060 & $-0,052$ & & 0,045 & $-0,039$ & 0,047 \\
\hline \multirow[t]{2}{*}{ Serviços } & & $-0,051$ * & 0,005 & $-0,056$ & * & 0,003 & $-0,047$ & 0,004 \\
\hline & & Número & co de observa & $\begin{array}{l}\text { Breusch } \\
\text { Hausma } \\
\text { as }=280.1\end{array}$ & & $\begin{array}{l}\mathrm{an}=56,06 \\
=59,865 \\
\text { Júmero d }\end{array}$ & $\begin{array}{l}38 \\
\text { divíduos }=\end{array}$ & $=140.069$ \\
\hline
\end{tabular}

Fonte: Elaboração própria a partir de dados da RAISMIGRA-1999-2001.

* Significativo a $5 \%$. 
A forma funcional utilizada considera as covariadas de experiência, experiência ao quadrado, dummies de localização geográfica, dummies de setor de atividade (conforme as subatividades do IBGE). Como as variáveis explicativas de idade, idade ao quadrado, dummy de sexo e dummies de grau de instrução não variam no tempo, elas são excluídas na análise de efeitos fixos.

No que se refere aos diferenciais salariais decorrentes da mobilidade inter-regional, a renda dos trabalhadores que se moveram para outras regiões de MinasGerais foi, em geral, menor que a renda dos trabalhadores que não-migraram. As maiores perdas foram verificadas para os trabalhadores que se moveram para o nordeste do Estado $(-24,8 \%)$, enquanto as menores foram para o sul de Minas (11,6\%). Na mobilidade interestadual, todos os diferenciais salariais registrados foram positivos. São Paulo e o Nordeste foram as regiões que registraram os maiores diferenciais salariais, 16,6\% e 11,9\%, respectivamente, seguidos do Rio de Janeiro, com 9,6\%. Cabe salientar que as dummies da região Norte, do Estado do Espírito Santo, da região Sul e da região Centro-Oeste não foram significativas.

Na mobilidade intersetorial, o trabalhador que mudou da indústria de transformação para a indústria extrativa mineral aumentou a sua renda em $15,1 \%$. Os trabalhadores que mudaram para outros setores apresentaram uma renda menor do que os trabalhadores que não mudaram. ${ }^{8}$ Apenas os serviços industriais de utilidade pública não foram significativos a $5 \%$.

A comparação dos resultados obtidos pelo método de mínimos quadrados agrupados (primeira coluna da Tabela 4) com a regressão de efeitos fixos (terceira coluna) evidencia importantes características sobre a heterogeneidade não observada existente. A inclusão dos efeitos fixos individuais reduz significativamente o tamanho dos coeficientes das dummies regionais e setoriais. Por exemplo, enquanto os diferenciais salariais para São Paulo ficaram em 43,5\% na regressão de MQO agrupado, na regressão de efeitos fixos estes diferenciais foram de apenas $16,6 \%$. No caso do Rio de Janeiro, esta diminuição dos coeficientes é ainda maior, passando de 40,7\% para 9,6\%. O mesmo ocorre quando consideramos os coeficientes das dummies de setores de atividade que, em geral, também se reduzem. Na administração pública, por exemplo, a redução salarial passa de $26,2 \%$ para $3 \%$. No comércio, a redução também é

8 Uma questão interessante a ser considerada nesta análise envolve a decisão de saída do emprego por iniciativa do trabalhador. Esta informação pode ser captada pela variável causa do desligamento e foi testada por meio de uma regressão de diferenciais salariais considerando apenas estes trabalhadores. Ou seja, usamos o modelo de efeitos fixos para estimar os diferenciais salariais dos trabalhadores que se desligaram do vínculo empregatício por iniciativa própria. Os resultados, contudo, não são conclusivos devido ao número insuficiente de observaçôes para a variável causa do desligamento. Vale a pena ressaltar que é preciso uma amostra muito grande, porquanto o número de migraçóes intersetoriais e inter-regionais não é tão grande assim. 
verificada, passando de 20,9\% para apenas 6,6\%. O destaque é a indústria extrativa mineral, cujos diferenciais salariais passam de $11,6 \%$ para $15,1 \%$.

No que se refere à experiência dos trabalhadores, o retorno desta sobre a renda é alto e cresce a taxas decrescentes. A dummy de ano mostra que 21,0 é o porcentual de ganhos salariais referentes ao ano de 2001.

Cabe ressaltar que não estamos considerando os preços relativos entre estas regióes. Isto significa que, em termos reais, tais resultados devem estar distorcidos pelas diferenças de custo de vida. (Azzoni, Carmo e Menezes, 2000 e 2003). Todavia, a disponibilidade de informações de preços é restrita para a região metropolitana e, de forma agregada, para áreas rurais e urbanas do Nordeste e Sudeste, até o momento.

De um modo geral, observando os diferenciais de renda obtidos pelo método de efeitos fixos, notamos que estes são bastante inferiores ao de MQO agrupado, podendo indicar um viés das estimativas provocado pelos efeitos não observáveis referentes ao trabalhador. Neste sentido, o principal efeito do modelo de efeitos fixos em relação ao de mínimos quadrados agrupados é a redução do tamanho dos coeficientes, o que demonstra que grande parte da variação salarial interindustrial e inter-regional pode ser atribuída às características individuais (que não variam no tempo).

É importante mencionar que, apesar da redução na magnitude e significância dos diferenciais salariais intersetoriais estimados, ainda constatou-se a existência de diferenças nos salários entre indústrias. Por exemplo, os trabalhadores da agropecuária recebem $10 \%$ a menos em relação ao trabalhador que permaneceu na indústria de transformação. Um argumento possível é que esses diferenciais são evidências de pressões específicas da indústria na determinação dos salários. No entanto, dado que as estimativas de efeitos fixos dos diferenciais interindustriais refletem somente a variação salarial dos indivíduos que se moveram da indústria de transformação de Minas Gerais, uma explicação alternativa para os diferenciais salariais interindustriais remanescentes é que existem habilidades individuais não mensuradas que não são fixas (mas que são eliminadas pela transformação within), porém diferem de acordo com o trabalho que o indivíduo está realizando.

Todavia, o resultado mais relevante deste estudo é que as diferenças específicas das indústrias e das regiões são de menor magnitude que o resultado inicialmente obtido por MQO. De fato, grande parte da variação nos salários previamente atribuída aos diferenciais interindustriais é realmente um reflexo das diferenças não observadas entre indivíduos que não poderiam ser eliminados numa estimação de equações de salário 
padrão de MQO agrupado. Um contra-argumento plausível é que nossos resultados também poderiam ser explicados pelos efeitos fixos não observados da firma, tendo em vista que as características observadas e não observadas são altamente correlacionadas no modelo de efeitos fixos. Porém, Abowd e Kramarz (1999), usando dados de matching entre empresa e trabalhador, demonstram que cerca de $90 \%$ dos diferenciais interindustriais podem ser explicados pelos efeitos fixos do indivíduo, ao passo que os efeitos da firma parecem explicar muito pouco dos diferenciais observados. Assim, podemos supor que a interpretação dos resultados de que existe um significativo papel da heterogeneidade individual não observada em vez da heterogeneidade não observada da firma é válida na explicação dos diferenciais salariais interindustriais.

Tendo em vista que os efeitos fixos captam a contribuição das características observadas invariantes no tempo (tais como sexo e educação, já que esta última varia muito pouco entre os anos de 1999 e 2001), bem como a heterogeneidade não observada, a comparação acima entre MQO agrupado e os resultados de efeitos fixos não é estritamente precisa. Por isso, reestimamos o modelo de MQO agrupado incluindo dummies individuais que captam a heterogeneidade não observada. Este exercício com os dados tem, portanto, o objetivo de comparar o poder de explicação das regressões com e sem as variáveis dummy de efeitos individuais (Tabela 5).

Devido ao grande número de observações, criamos uma subamostra9 que foi utilizada para ambas as estimações. Os resultados evidenciam uma elevada proporção da variação nos salários entre indivíduos, que passa a ser explicada quando incluímos os efeitos fixos. Enquanto o $\mathrm{R}^{2}$ ajustado do modelo de $\mathrm{MQO}$ agrupado sem dummies individuais é de 21\%, o $\mathrm{R}^{2}$ ajustado da regressão que inclui estas dummies é de $81 \%$, indicando que existe apenas uma pequena variação residual nos salários que não pode ser atribuída às características observadas e não observadas dos indivíduos. Portanto, a maior parte da variação nos salários pode ser atribuída diretamente a diferenças entre os indivíduos.

9 A subamostra possui 2.887 indivíduos em cada ano, perfazendo um total de 5.774 observações, equivalente a $2 \%$ do total da amostra. 
TABELA 5 - DIFERENCIAIS DE RENDA DOS TRABALHADORES - VARIÁVEL DEPENDENTE: LOGARITMO DA RENDA

\begin{tabular}{|c|c|c|c|c|c|}
\hline & \multicolumn{2}{|c|}{ MQO agrupado } & \multicolumn{2}{|c|}{ MQO agrupado } \\
\hline & & \multicolumn{2}{|c|}{ com dummies de indivíduo** } & \multicolumn{2}{|c|}{ sem dummies de indivíduo } \\
\hline & & Coef. & Erro padrão & Coef. & Erro padrãc \\
\hline \multicolumn{2}{|l|}{ Constante } & 8,724 * & 0,353 & 5,802 * & 0,018 \\
\hline \multicolumn{2}{|c|}{ Ano 2001} & 0,226 * & 0,011 & 0,175 * & 0,021 \\
\hline \multicolumn{6}{|c|}{ Características do trabalhador } \\
\hline \multicolumn{2}{|c|}{ Experiência } & 0,005 * & 0,001 & 0,008 * & 0,000 \\
\hline \multicolumn{2}{|c|}{ Experiência $^{2}$} & 0,000 * & 0,000 & 0,000 * & 0,000 \\
\hline \multicolumn{2}{|c|}{$\begin{array}{l}\text { Localização } \\
\text { Região Metropolitana BH }\end{array}$} & \multicolumn{2}{|c|}{ (excluída) } & \multicolumn{2}{|c|}{ (excluída) } \\
\hline \multirow[t]{6}{*}{ Interior de MG } & Norte de MG & $-0,167$ & 0,285 & 0,452 & 0,411 \\
\hline & Nordeste de MG & $-0,904$ * & 0,285 & $-0,175$ & 0,411 \\
\hline & Sudeste de MG & $-0,025$ & 0,120 & $-0,183$ & 0,173 \\
\hline & Sul de MG & $-0,303$ & 0,291 & $-0,091$ & 0,418 \\
\hline & Triângulo mineiro & $-0,195$ & 0,247 & 0,999 * & 0,356 \\
\hline & Centro de MG & $-0,102$ & 0,092 & $-0,078$ & 0,133 \\
\hline \multicolumn{2}{|l|}{ Região Norte } & 0,401 & 0,494 & $-0,204$ & 0,711 \\
\hline \multicolumn{2}{|c|}{ Região Nordeste } & $-0,148$ & 0,182 & $-0,114$ & 0,262 \\
\hline \multicolumn{2}{|c|}{ Espírito Santo } & 0,142 & 0,497 & $-0,262$ & 0,715 \\
\hline \multicolumn{2}{|l|}{ Rio de Janeiro } & 0,617 * & 0,202 & 0,855 * & 0,291 \\
\hline \multicolumn{2}{|l|}{ São Paulo } & 0,106 & 0,081 & 0,773 * & 0,117 \\
\hline \multicolumn{2}{|c|}{ Região Sul } & 0,365 * & 0,175 & 0,735 * & 0,252 \\
\hline \multicolumn{2}{|c|}{ Região Centro Oeste } & 0,178 & 0,157 & 0,535 * & 0,226 \\
\hline \multicolumn{6}{|c|}{ Setor de atividade } \\
\hline \multicolumn{2}{|c|}{ Adm. Pública } & 0,315 * & 0,119 & 0,103 & 0,171 \\
\hline \multicolumn{2}{|l|}{ Agropecuária } & 0,247 & 0,151 & $-0,088$ & 0,217 \\
\hline \multicolumn{2}{|l|}{ Comércio } & $-0,056$ & 0,035 & $-0,228$ * & 0,051 \\
\hline \multicolumn{2}{|c|}{ Construção Civil } & $-0,054$ & 0,059 & $-0,114$ & 0,085 \\
\hline \multicolumn{2}{|c|}{ Extrativa Mineral } & 0,190 & 0,178 & 0,591 * & 0,254 \\
\hline \multicolumn{2}{|c|}{ Ind. Transformação } & \multicolumn{2}{|c|}{ (excluída) } & \multicolumn{2}{|c|}{ (excluída) } \\
\hline SIUP & & 0,478 & 0,361 & 0,250 & 0,519 \\
\hline Serviços & & $-0,041$ & 0,030 & 0,078 & 0,043 \\
\hline $\mathrm{N}^{\circ}$ Observaçõe & & & 5.774 & & 5.774 \\
\hline R2 & & & 0,905 & & 0,211 \\
\hline R2 ajustado & & & 0,809 & & 0,208 \\
\hline
\end{tabular}

Fonte: Elaboração própria a partir de dados da RAISMIGRA-1999-2001

* Significativo a $5 \%$.

** Dummies individuais para os 2.887 indivíduos da subamostra foram omitidas. 


\section{CONCLUSÃO}

Este trabalho teve como objetivo a verificação dos diferenciais salariais intersetoriais e inter-regionais dos trabalhadores da indústria de transformação mineira entre 1999 e 2001, controlando pelas características observáveis e não observáveis destes indivíduos. Os principais resultados obtidos indicam que ao considerarmos as características não observáveis dos trabalhadores - tais como habilidade, motivação, entre outras - as diferenças específicas dos setores e das regiões são de menor magnitude que os resultados inicialmente verificados por MQO. De fato, grande parte da variação nos salários previamente atribuída aos diferenciais interindustriais é realmente um reflexo das diferenças não observadas entre indivíduos que não poderiam ser eliminados numa estimação de equações de salário padrão de MQO agrupado. Uma possível conseqüência deste resultado é que os efeitos específicos da indústria têm uma função mais limitada na explicação da estrutura salarial industrial.

Apesar da redução na magnitude e significância dos diferenciais salariais interindustriais e inter-regionais estimados, ainda verificamos a existência de diferenças nos salários entre indústrias e regiões. Além disso, existe uma elevada proporção da variação nos salários entre indivíduos que passa a ser explicada com a metodologia de efeitos fixos. Utilizando uma subamostra aleatória de $2 \%$ do total, o $\mathrm{R}^{2}$ ajustado do modelo de MQO agrupado sem efeitos fixos é de $21 \%$, ao passo que o $\mathrm{R}^{2}$ ajustado da regressão que inclui dummies individuais é de $81 \%$. Isto indica que existe apenas uma pequena variação residual nos salários que não pode ser atribuída às características observadas e não observadas dos indivíduos. Assim, a maior parte da variação nos salários pode ser atribuída diretamente a diferenças entre os indivíduos.

\section{REFEREANCIAS BIBLIOGRÁFICAS}

Abowd, J. M.; Kramarz, F. The analysis of labor markets using matched employeremployee data. In: Ashenfelter, O.; Card, D. (eds.), Handbook of labor economics. Elsevier Science B. V., p. 2629-2711, 1999.

Angrist, J.; Krueger, A. Empirical strategies in labor economics. In: Ashenfelter, O.; Card, D. (eds.), Handbook of labor economics, v. 3A, Elsevier, 1999.

Arbache, J. S.; De Negri, J. A. Filiação industrial e diferencial de salários no Brasil. Revista Brasileira de Economia, Rio de Janeiro, v. 58, n. 2, p. 159-184, 2004.

Arbache, J. S. Wage differentials in Brazil: theory and evidence. Journal of Development Studies, Londres, v. 38, n. 2, p. 691-714, 2001.

Azzoni, C. R.; Carmo, H. E.; Menezes, T. Comparações da paridade do poder de compra entre cidades: aspectos metodológicos e aplicação ao caso brasileiro. Pesquisa e Planejamento Econômico, Rio de Janeiro, v. 33, n. 1, 2003. 
. Índice de custo de vida comparativo para as principais regiões metropolitanas brasileiras: 1981-1999. Estudos Econômicos, São Paulo, v. 30, n. 1, p. 165-186, jan./mar. 2000.

Azzoni, C. R.; Menezes, T. A. Convergência de renda real e nominal entre as regiões metropolitanas brasileiras: uma análise de dados de painel. In: Anais do XXVIII Encontro de Economia. Campinas, dezembro de 2000.

Azzoni, C. R.; Servo, L. M. S. Education, cost of living and regional wage inequality in Brazil in the 90's. Papers in Regional Science, Alemanha, v. 81, p. 157-175, 2002.

Carruth, A.; Collier, W.; Dickerson, A. Inter-industry wage differences and individual heterogeneity. Oxford Bulletin of Economics and Statistics, Oxford, v. 66, n. 5, 2004 .

Corseuil, C. H.; Santos, D. D. Fatores que determinam o nível salarial no setor formal brasileiro. In: Corseuil, C. H. et al. (orgs.), Estrutura salarial: aspectos conceituais e novos resultados para o Brasil. Rio de Janeiro: IPEA, 2002.

Dickens, W. T.; Katz, L. F. Inter-industry wage differences and theory of wage determination. NBER Working Paper n. 2271, 1987.

Fernandes, R. Desigualdade salarial: aspectos teóricos. In: Corseuil, C. H. et al. (orgs.), Estrutura salarial: aspectos conceituais e novos resultados para o Brasil. Rio de Janeiro: IPEA, 2002.

Gittleman, M.; Wolff, E. N. International comparisons of inter-industry wage differentials. Review of Income and Wealth, v. 39, p. 295-312, 1993.

Kahn, L. M. Collective bargaining and the inter-industry wage structure: international evidence. Economica, v. 65, p. 507-534, 1998.

Krueger, A. B.; Summers, L. H. Efficiency wages and the inter-industry wage structure. Econometrica, 56, p. 259-293, 1988.

Menezes-Filho, N. Equações de rendimentos: questões metodológicas. In: Corseuil, C. H. et al. (orgs.), Estrutura salarial: aspectos conceituais e novos resultados para o Brasil. Rio de Janeiro: IPEA, 2002.

. Microeconometria. In: Lisboa, M.; Menezes-Filho, N. (orgs.), Microeconomia e sociedade no Brasil. Rio de Janeiro: Editora Contra-capa, 2001.

Ministério do Trabalho e Emprego. Raismigra. Brasília: MTE, 1999/2001.

. Raismigra: modelos painel e vínculo - orientações para uso. 02/10/2003. Mimeografado

Wooldridge, J. M. Econometric analysis of cross-section and panel data. Massachusetts: MIT, 2002. 